\title{
Accounting for the analytical properties of the quark propagator from Dyson-Schwinger equation
}

\author{
S. M. Dorkin,,${ }^{1,2}$ L. P. Kaptari, ${ }^{1,3}$ and B. Kämpfer ${ }^{3,4}$ \\ ${ }^{1}$ Bogoliubov Lab. Theor. Phys., 141980, JINR, Dubna, Russia \\ ${ }^{2}$ International University Dubna, Dubna, Russia \\ ${ }^{3}$ Helmholtz-Zentrum Dresden-Rossendorf, \\ PF 510119, 01314 Dresden, Germany \\ ${ }^{4}$ Institut für Theoretische Physik, TU Dresden, 01062 Dresden, Germany
}

\begin{abstract}
An approach based on combined solutions of the Bethe-Salpeter (BS) and Dyson-Schwinger (DS) equations within the ladder-rainbow approximation in the presence of singularities is proposed to describe the meson spectrum as quark-antiquark bound states. We consistently implement into the BS equation the quark propagator functions from the DS equation, with and without pole-like singularities, and show that, by knowing the precise positions of the poles and their residues, one is able to develop reliable methods of obtaining finite interaction BS kernels and to solve the BS equation numerically. We show that, for bound states with masses $M<1 \mathrm{GeV}$, there are no singularities in the propagator functions when employing the infrared part of the Maris-Tandy kernel in truncated BS-DS equations. For $M>1 \mathrm{GeV}$, however, the propagator functions reveal pole-like structures. Consequently, for each type of mesons (unflavored, strange and charmed) we analyze the relevant intervals of $M$ where the pole-like singularities of the corresponding quark propagator influence the solution of the BS equation and develop a framework within which they can be consistently accounted for. The BS equation is solved for pseudo-scalar and vector mesons. Results are in a good agreement with experimental data. Our analysis is directly related to the future physics programme at FAIR with respect to open charm degrees of freedom.
\end{abstract}




\section{INTRODUCTION}

The investigation of mesons as bound states of quarks is of fundamental interest for understanding the low-energy degrees of freedom of strong interaction and its relation to QCD. It is tightly connected with non perturbative methods in QCD and directly related to study of such important phenomena as dynamical chiral symmetry breaking, confinement of quarks, mass splitting of meson multiplets etc. In principle, lattice QCD simulations can provide "experimental" information on most non-perturbative effects of QCD. However, with respect to some practical limitations in lattice calculations, it is extremely important to elaborate in parallel reliable phenomenological or semi-phenomenological approaches to describe the main features of non-perturbative QCD. Such approaches would allow one to extend calculations to large distances, nowadays inaccessible for exact calculations. From the other side, the elaboration of consistent models for QCD bound states in vacuum can serve as clue in understanding the in-medium properties of hadrons at high densities and temperatures. For instance, the planned experiments at FAIR, GSI [1, 2] and NICA [3], offer the next-generation investigations of charmed probes in proton and anti-proton induced reactions at nuclei as well as heavy-ion collisions accessing the maximum baryon density region. In contrast, the running experiments at RHIC and LHC address, among other important issues, the behaviour of charmed probes in hot matter in the deconfinement region. An ultimate prerequisite of the interpretation of current and future experiments including high-statistic charmed probes is the firm theoretical understanding of the meson spectrum in such a mass range. Once this is accomplished one goes ahead towards in medium-effect by appropriate methods.

In the present paper, the mesonic bound states in vacuum are described within the framework of the homogeneous Bethe-Salpeter (BS) equation [4] with momentum dependent quark mass functions, determined by the Dyson-Schwinger (DS) equation. For the sake of consistency, in both, the BS and DS equations one uses identical interaction kernels. In principle, if one were able to solve the complete set of Dyson-Schwinger equations for quark and gluon propagators and vertex functions as well, the approach would not depend on additional parameters. However, due to known difficulties, in real calculations one restricts oneself to first, one-loop term of the perturbative series and, based on the obtained results, establish a general form of the phenomenological gluon propagators to be used in DS and BS equations. In elaborating such approaches it is important that the suggested BS kernels, which implicitly is also contained 
in the DS equation, and dressed quark-gluon vertices, pertaining both BS and DS equations, to be consistent to each other, i.e. to guarantee at least the Ward-Takahshi identity [5]. One of such an approach is known as Maris-Tandy model [6] which is based on the rainbow-ladder approximation of the DS equation, see also [7]. The merit of the approach is that, once the effective parameters are fixed, the spectrum of known mesons is supposed to be described on the same footing, including also excited states. The model has been applied to explain successfully many spectroscopic data, such as meson masses [6, 8-12], electromagnetic properties of pseudoscalar mesons and their radial excitations [13] and other observables [14 20].

The fact that the model encounters difficulties in describing heavy mesons, $M>1 \mathrm{GeV}$, with at least one light $(\mathrm{u}, \mathrm{d}$ or $\mathrm{s})$ quark is not often mentioned. However, now this is not a critical defect of the model since the source of difficulties seems to be firmly understood - these are pole-like singularities in the propagator functions from the DS equation at large meson masses [21]. An accurate treatment of the singularities relevant to the kinematical region of the BS equation can essentially facilitate computations of the BS kernel removing in such a way the difficulties and allowing to obtain stable results.

In the present paper we continue our investigation [21] of the prerequisites to the interaction kernel of the combined Dyson-Schwinger and Bethe-Salpeter formalisms to describe the meson mass spectrum including heavier mesons and excited states. In the previous paper [21] it was argued that the propagator functions from the DS equation are not analytical functions in the Euclidean complex plane impeding numerical solutions of the BS equation. Our goal herein is to analyze the analytical properties of the quark propagators in the whole kinematical region relevant to light and semi-heavy meson masses, supply a method to implement the singular propagators in solving numerically the BS equation and solve the BS equation for mesons in the presence of singularities. To this end we solve the DS equation in the rainbow ladder approximation by making use of the hyperspherical harmonics basis to decompose the propagators and the corresponding potential and solve numerically the resulting DS equations for the coefficients of such a decomposition. Then, the further analysis of quark singularities is based on a combined application of Rouché's theorem and a graphical representation of the inverse propagators as vortex fields of the corresponding complex functions, see Ref. [21] for details. In the present paper we restrict ourselves to meson masses up to $M=3.5 \mathrm{GeV}$ and, correspondingly, investigate all the relevant poles of quarks of different flavors $(\mathrm{u}, \mathrm{d}, \mathrm{s}$ and c quarks) solely in this kinematical region. Then we suggest a method of accounting for 
singularities and solve the BS equation for light and heavier mesons.

It should be noted that there exist other approaches based on the same physical ideas of exploring effective quark interactions. For instance, in Ref. [22] (and references therein quoted) it was demonstrated that within an approach with instanton fluctuations of the QCD vacuum it is possible to describe the mechanism of formation of mesons as bound states of quarks and to analyse their main physical properties, including the relation between quark propagators and quark condensates. Other approaches employ simpler interactions, e.g. a separable interaction for the effective coupling [17]. Such models describe also fairly well properties of light mesons, nevertheless, the investigation of heavier mesons and excited states, consisting even of light ( $u$, $\mathrm{d}$ and s) quarks, requires implementations of more accurate numerical methods to solve the corresponding equations.

Our paper is organized as follows. In Secs. II, IIB, IIC and III we briefly discuss the truncated BS and DS equations within the rainbow approximation relevant to describe the mesons as quark-antiquark bound states. The domain of the complex plane of Euclidean momenta, where the solutions of the BS equation are sought, and the corresponding propagator

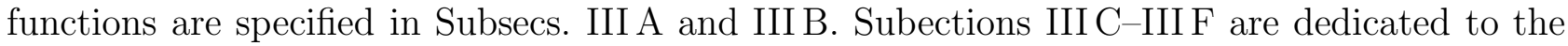
solution of the truncated DS equation for complex momenta and to a thorough analysis of the singularities of the propagator functions in the domain of the Euclidean space relevant to BS solutions for mesons with masses up to $3.5 \mathrm{GeV}$. Convenient parametrizations for the propagator functions of $\mathrm{u}, \mathrm{d}, \mathrm{s}$ and $\mathrm{c}$ quarks used to determine the BS kernel in the domain of its analyticity are presented in Subsec. IIID, The subsequent Sections are aimed at investigations of methods of solving the BS equation in presence of singularities. In Subsec. IVA we present results of numerical calculations of the mass spectra of the pseudo-scalar mesons. Alternative numerical approaches and nuances in solving the BS equation for heavy mesons with quarks of equal masses are briefly discussed in Subsecs. IVC, IVD and IVE. Summary and conclusions are collected in Sec. V] In the Appendix some useful relations used in solving the BS equation are presented. 


\section{BETHE-SALPETER EQUATION}

\section{A. Ladder-rainbow approximation}

To determine the bound-state energy (mass) of a quark-antiquark pair one needs to solve the Bethe-Salpeter equation, which in the ladder approximation (hereafter referred to as truncated Bethe-Salpeter (tBS) equation) and in Euclidean space, reads

$$
\Gamma(P, p)=-\frac{4}{3} \int \frac{d^{4} k}{(2 \pi)^{4}} \gamma_{\mu} S\left(\frac{1}{2} P+k\right) \Gamma(P, k) S\left(-\frac{1}{2} P+k\right) \gamma_{\nu}\left[g^{2} \mathcal{D}_{\mu \nu}(p-k)\right],
$$

where the interaction kernel $g^{2} \mathcal{D}_{\mu \nu}\left(k^{2}\right) \equiv \mathcal{D}\left(k^{2}\right) d_{\mu \nu}\left(k^{2}\right)$ is chosen in the Landau gauge, $d_{\mu \nu}\left(k^{2}\right)=$ $\left[\delta_{\mu \nu}-k_{\mu} k_{\nu} / k^{2}\right], \Gamma(P, p)$ is the tBS vertex function, and $S\left(k_{1,2}\right)=-i \gamma \cdot k_{1,2} \sigma_{v}\left(k_{1,2}^{2}\right)+\sigma_{s}\left(k_{1,2}^{2}\right)$ are the quark propagators with the propagator functions $\sigma_{v, s}\left(k^{2}\right)$. The total $P$ and relative momenta $p$ are defined as $P=p_{1}+p_{2}$ and $p=\left(p_{1}-p_{2}\right) / 2$, where $p_{1,2}$ are the momenta of the constituent quarks. The two quarks interact via gluon exchange encoded in $\left[g^{2} \mathcal{D}_{\mu \nu}(p-k)\right]$. The vertex function $\Gamma(P, k)$ is a $4 \times 4$ matrix and, therefore, may contain 16 different functions.

The interaction kernel is chosen within the ladder rainbow approximation. It has been widely used to study the physics of dynamical chiral symmetry breaking [9], decay constants [6, 10, 11, 13] and other observables [14] and has been found to provide a good agreement with experimental data. The employed vertex-gluon kernel in the rainbow approximation is chosen here in the form [6, $9,18,20]$

$$
\mathcal{D}\left(k^{2}\right)=\left(\frac{4 \pi^{2} D k^{2}}{\omega^{2}} e^{-k^{2} / \omega^{2}}+\cdots\right)
$$

where the first term originates from the effective infrared (IR) part of the interaction determined by soft non-pertubative effects, while further ones (hidden in ...) ensure the correct ultraviolet (UV) asymptotic behaviour of the QCD running coupling. A detailed investigation of the interplay of IR and UV terms has shown [11, 23] that the IR part is dominant for light u, d and s quarks, with a decreasing role for heavier quark masses (c and b) for which the UV part may play a role in forming meson masses with $M>4 \mathrm{GeV}$. In the present paper we consider mesons with masses up to $3.5 \mathrm{GeV}$ and, consequently, in the interaction kernel $\mathcal{D}\left(k^{2}\right)$ the spelled out IR term is accounted for. It depends on two parameter, $D$ and $\omega$. Since within the ladder approximation the tBS amplitude does not depend on the total momentum $P$, in what follows in $\Gamma(P, p)$ and in all subsequent partial amplitudes we omit $P$ as redundant notation.

The general structure of vertex functions describing bound states of spinor particles has been investigated in detail, for example, in [24-26]. To release from the matrix structure, the vertex 
function $\Gamma$ is expanded into functions which in turn are determined by angular momentum and parity of the corresponding meson known as the spin-angular harmonics [14, 25, 27]:

$$
\Gamma(p)=\sum_{\alpha} g_{\alpha}(p) \mathcal{T}_{\alpha}(\boldsymbol{p}), \quad g_{\alpha}(p)=\int d \Omega \boldsymbol{p} \operatorname{Tr}\left[\Gamma(p) \mathcal{T}_{\alpha}^{+}(\boldsymbol{p})\right] .
$$

The complete set of spin-angular matrices are chosen as:

(i) in the ${ }^{1} S_{0}$ pseudo-scalar channel

$$
\begin{aligned}
\mathcal{T}_{1}(\boldsymbol{p})=\frac{1}{\sqrt{16 \pi}} \gamma_{5}, & \mathcal{T}_{2}(\boldsymbol{p})=\frac{1}{\sqrt{16 \pi}} \gamma_{0} \gamma_{5}, \\
\mathcal{T}_{3}(\boldsymbol{p})=-\frac{1}{\sqrt{16 \pi}} \widehat{n}_{\mathbf{p}} \gamma_{0} \gamma_{5}, & \mathcal{T}_{4}(\boldsymbol{p})=-\frac{1}{\sqrt{16 \pi}} \widehat{n}_{\mathbf{p}} \gamma_{5},
\end{aligned}
$$

and (ii) for the ${ }^{3} S_{1}-^{3} D_{1}$ vector channel

$$
\begin{array}{rlrl}
\mathcal{T}_{1}(\boldsymbol{p}) & =\sqrt{\frac{1}{16 \pi}} \widehat{\xi}_{\mathcal{M}}, & \mathcal{T}_{2}(\boldsymbol{p}) & =-\sqrt{\frac{1}{16 \pi}} \gamma_{0} \widehat{\xi}_{\mathcal{M}}, \\
\mathcal{T}_{3}(\boldsymbol{p}) & =-\sqrt{\frac{3}{16 \pi}}\left(n_{\mathbf{p}} \xi_{\mathcal{M}}\right), & \mathcal{T}_{4}(\boldsymbol{p}) & =\sqrt{\frac{3}{32 \pi}} \gamma_{0}\left[-\left(n_{\mathbf{p}} \xi_{\mathcal{M}}\right)+\hat{n}_{\mathbf{p}} \widehat{\xi}_{\mathcal{M}}\right], \\
\mathcal{T}_{5}(\boldsymbol{p}) & =\sqrt{\frac{1}{32 \pi}}\left[\widehat{\xi}_{\mathcal{M}}+3\left(n_{\mathbf{p}} \xi_{\mathcal{M}}\right) \widehat{n}_{\mathbf{p}}\right], \mathcal{T}_{6}(\boldsymbol{p})=\sqrt{\frac{1}{32 \pi}} \gamma_{0}\left[\widehat{\xi}_{\mathcal{M}}+3\left(n_{\mathbf{p}} \xi_{\mathcal{M}}\right) \widehat{n}_{\mathbf{p}}\right], \\
\mathcal{T}_{7}(\boldsymbol{p}) & =-\sqrt{\frac{3}{16 \pi}} \gamma_{0}\left(n_{\mathbf{p}} \xi_{\mathcal{M}}\right), & \mathcal{T}_{8}(\boldsymbol{p}) & =\sqrt{\frac{3}{32 \pi}}\left[-\left(n_{\mathbf{p}} \xi_{\mathcal{M}}\right)+\hat{n}_{\mathbf{p}} \widehat{\xi}_{\mathcal{M}}\right],
\end{array}
$$

where all the above scalar products are written in Minkowski space and the unit vector $n_{\mathbf{p}}$ is defined as $n_{\mathbf{p}}=(0, \mathbf{p} /|\mathbf{p}|)$. The left hand side of (5) depends implicitly on $\mathcal{M}$, which denotes the components of the polarization vector $\xi_{\mathcal{M}} \equiv\left(0, \boldsymbol{\xi}_{\mathcal{M}}\right)$ fixed by $\boldsymbol{\xi}_{+1}=-(1, i, 0) / \sqrt{2}$, $\boldsymbol{\xi}_{-1}=(1,-i, 0) / \sqrt{2}, \boldsymbol{\xi}_{0}=(0,0,1)$. Similar complete sets of spin-angular harmonics have been employed also in Refs. [18, 26].

With Eqs. (3)-(15) the integral matrix form of the BS equation (1) can be reduced to a system of two-dimensional integral equations with respect to the partial vertices $g_{\alpha}(p)$, cf. [25, 27].

\section{B. The hyperspherical decomposition}

To further reduce the dimension of the integral we decompose, in Euclidean space, the partial vertices $g_{\alpha}(p)$ and the interaction kernel $\mathcal{D}(p-k)$ in (11) over the basis of spherical harmonics $\mathrm{Y}_{\operatorname{lm}}(\theta, \phi)$ and normalized Gegenbauer polynomials $X_{n l}(\chi)$, i.e. we use the hyperharmonic basis

$$
Z_{n l m}(\chi)=X_{n l}(\chi) \mathrm{Y}_{\operatorname{lm}}(\theta, \phi) \equiv \sqrt{\frac{2^{2 l+1}}{\pi} \frac{(n+1)(n-l) ! l !^{2}}{(n+l+1) !}} \sin ^{l} \chi G_{n-l}^{l+1}(\cos \chi) \mathrm{Y}_{\operatorname{lm}}(\theta, \phi)
$$


where $G_{n-l}^{l+1}(\cos \chi)$ are the Gegenbauer polynomials of the hyperangle $\chi$ with $\cos \chi=\frac{p_{4}}{\tilde{p}}$ and $\sin \chi=\frac{|\mathbf{p}|}{\tilde{p}}$ and $\tilde{p}=\sqrt{p_{4}^{2}+\boldsymbol{p}^{2}}$ of an Euclidean 4-vector $p$. Then, the partial decomposition of the vertex functions $g_{\alpha}\left(p_{4}, \boldsymbol{p}\right)(\alpha=1 \ldots 4$ and $\alpha=1 \ldots 8$ for pseudoscalar and vector mesons, respectively) and interaction kernel reads

$$
\begin{aligned}
& g_{\alpha}\left(p_{4}, \boldsymbol{p}\right)=\sum_{n l_{\alpha}} \varphi_{\alpha, l_{\alpha}}^{n}(\tilde{p}) X_{n l_{\alpha}}\left(\chi_{p}\right) \mathcal{T}_{\alpha}(\boldsymbol{p}), \\
& \mathcal{D}(p-k)=2 \pi^{2} \sum_{\kappa \lambda \mu} \frac{1}{\kappa+1} V_{\kappa}(\tilde{p}, \tilde{k}) X_{\kappa \lambda}\left(\chi_{p}\right) X_{\kappa \lambda}\left(\chi_{k}\right) Y_{\lambda \mu}\left(\Omega_{p}\right) Y_{\lambda \mu}^{*}\left(\Omega_{k}\right) .
\end{aligned}
$$

We label here and in the following the modulus of an Euclidean vector $p=\left(p_{4}, \boldsymbol{p}\right)$ by a tilde, i.e. $\tilde{p} \equiv \sqrt{p_{4}^{2}+\boldsymbol{p}^{2}}$. Actually, in Eq. (77) the summation over $l_{\alpha}$ is restricted by the corresponding orbital momentum encoded in the spin-angular matrices $\mathcal{T}_{\alpha}(\boldsymbol{p})$. It can be seen from Eq. (44) that the spin-angular harmonics $\mathcal{T}_{1,2}(\boldsymbol{p})$ in the ${ }^{1} S_{0}$ channel (pseudoscalar mesons) carry the momentum $l_{\alpha}=0$, while for $\mathcal{T}_{3,4}(\boldsymbol{p})$ one has $l_{\alpha}=1$. Analogously for vector mesons (cf. Eq. (15) ) $l_{\alpha}=0$ in $\mathcal{T}_{1,2}(\boldsymbol{p}), l_{\alpha}=2$ in $\mathcal{T}_{5,6}(\boldsymbol{p})$ and $l_{\alpha}=1$ otherwise.

Changing the integration variables to the hyperspace, $d^{4} k=\tilde{k}^{3} \sin ^{2} \chi_{k} \sin \theta_{k} d \tilde{k} d \chi_{k} d \theta_{k} d \phi_{k}$, inserting (8) and (17) into (11) and performing the necessary angular integrations we obtain a system of integral equations for the expansion coefficients $\varphi_{\alpha, l_{\alpha}}^{n}$ :

$$
\varphi_{\alpha, l_{\alpha}}^{n}(\tilde{p})=\sum_{\beta} \sum_{m=1}^{\infty} \int d \tilde{k} \tilde{k}^{3} S_{\alpha \beta}(\tilde{p}, \tilde{k}, m, n) \varphi_{\beta, l_{\beta}}^{m}(\tilde{k}) .
$$

The explicit expressions for the coefficients $S_{\alpha \beta}(\tilde{p}, \tilde{k}, m)$ result from the corresponding angular integrations over $d \Omega_{\mathbf{p}}, d \Omega_{\mathbf{k}}, \sin ^{2} \chi_{k} d \chi_{k}$ and $\sin ^{2} \chi_{p} d \chi_{p}$ which can be written in the form

$$
S_{\alpha \beta}(\tilde{p}, \tilde{k}, m, n)=\sum_{\kappa} \int \sin ^{2} \chi_{k} d \chi_{k} X_{m l_{\beta}}\left(\chi_{k}\right) X_{\kappa \lambda}\left(\chi_{k}\right) \sigma_{s, v}\left(k_{1}^{2}\right) \sigma_{s, v}\left(k_{2}^{2}\right) A_{\alpha \beta}\left(\tilde{p}, \tilde{k}, \kappa, \chi_{k}, n\right),(10
$$

where

$$
k_{1,2}^{2}=\left(\frac{1}{2} P \pm k\right)^{2}=-\frac{1}{4} M^{2}+\tilde{k}^{2} \pm i M \tilde{k} \cos \chi_{k}
$$

with $k$ as the relative momentum of two quarks, and the total momentum $P=(i M, \mathbf{0})$. The quantity $A_{\alpha \beta}$ resulting from evaluations of traces and angular integration in the 3-momentum space, has schematically the form

$$
\begin{aligned}
& A_{\alpha \beta}\left(\tilde{p}, \tilde{k}, \kappa, \chi_{k}\right) \simeq \int d \Omega_{\boldsymbol{p}} d \Omega_{\boldsymbol{k}} \sin ^{2} \chi_{p} d \chi_{p} V_{\kappa}(\tilde{p}, \tilde{k}) d_{\mu \nu}\left((p-k)^{2}\right) \times \\
& X_{n l_{\alpha}}\left(\chi_{p}\right) X_{\kappa \lambda}\left(\chi_{p}\right) \mathrm{Y}_{\lambda \mu}\left(\theta_{p}, \phi_{p}\right) \mathrm{Y}_{\lambda \mu}^{*}\left(\theta_{k}, \phi_{k}\right) \operatorname{Tr}\left[\gamma_{\mu} \ldots \mathcal{T}_{\beta}(\boldsymbol{k}) \ldots \mathcal{T}_{\alpha}^{+}(\boldsymbol{p}) \gamma_{\mu}\right]
\end{aligned}
$$


The angular structure of the integrand in (12) is rather simple: it contains a series of products of spherical harmonics, Gegenbauer polynomials and scalar products of $(\boldsymbol{p} \boldsymbol{k}) \sim$ $\mathrm{Y}_{1 \mathrm{~m}}^{*}\left(\theta_{p}, \phi_{p}\right) \mathrm{Y}_{1 \mathrm{~m}}\left(\theta_{k}, \phi_{k}\right)$, i.e. all angular integrations over $d \Omega \boldsymbol{p} d \Omega \boldsymbol{k}^{d \chi_{p}}$ can be performed explicitly. These integrations provide a smooth quantity $A_{\alpha \beta}\left(\tilde{p}, \tilde{k}, \kappa, \chi_{k}\right)$ being free of any singularities. That means the analytical structure of the kernel (10) is entirely determined by the propagator functions $\sigma_{s, v}\left(\tilde{k}^{2}\right)$.

Recall that in Eqs. (9)-(12) the indices $(\alpha, \beta)$ label the tBS components in the spinor space ( $\alpha, \beta=1 \ldots 4$ for pseudoscalar mesons and $\alpha, \beta=1 \ldots 8$ for vector mesons), $(m, n)$ denote the number of terms in the Gegenbauer decomposition (7), and $l_{\alpha, \beta}$ are entirely determined by the corresponding components $\mathcal{T}_{\alpha, \beta}$ of the spin-angular basis.

\section{Numerical solutions}

Now we proceed with solving the BS equation (9) for the partial vertices $\varphi_{\alpha, l_{\alpha}}^{n}(\tilde{p})$. Equations (9)-(12) represent the desired system of the BS one-dimensional integral equations of the Fredholm type within the hyperspherical harmonics formalism to be solved numerically. Before choosing a specific computational algorithm one has to analyze at least the existence and uniqueness of the solution. Obviously, this issue is directly connected to the properties of the interaction kernels. As known, the main requirement for the existence of solutions of Fredholm type equations is the finiteness of the integral kernel, i.e. of the quantity $S_{\alpha \beta}(\tilde{p}, \tilde{k}, m, n)$ in our case. As mentioned above the function $A_{\alpha \beta}\left(\tilde{p}, \tilde{k}, \kappa, \chi_{k}\right)$, which determines the kernel $S_{\alpha \beta}(\tilde{p}, \tilde{k}, m, n)$, is finite by construction. That means the only source of troubles can appear from the propagator functions $\sigma_{s, v}\left(\tilde{k}^{2}\right)$ in (10), which, in turn, are the solution of the tDS equation with the same interaction kernel (2). Accordingly, prior to solve the tBS equation, firstly we shall proceed with an analysis of the propagator functions $\sigma_{s, v}\left(\tilde{k}^{2}\right)$ from the Dyson-Schwinger equation in Euclidean space.

\section{DYSON-SCHWINGER EQUATION}

\section{A. The relevant region for the truncated Dyson-Schwinger equation}

We are interested in the analytical structure of the propagator functions $\sigma_{s, v}\left(\tilde{k}^{2}\right)$ inside and in the neighbourhood of the complex momentum region in the Euclidean space dictated by the 
tBS equation (11). This momentum region is displayed as the dependence of the imaginary part of the quark momentum squared $\operatorname{Im} \tilde{k}^{2}$ on its real part Re $\tilde{k}^{2}$ from the tBS equation determining in Euclidean complex momentum plane a domain restricted by a parabola

$$
\operatorname{Im} \tilde{k}^{2}= \pm M \sqrt{\operatorname{Re} \tilde{k}^{2}+\frac{1}{4} M^{2}}
$$

with vertex at $\operatorname{Im} \tilde{k}^{2}=0$ at $\operatorname{Re} \tilde{k}^{2}=-M^{2} / 4$ depending on the meson mass $M$.

Note that regardless of the form of the interaction kernel, the investigation of the analytical structure of the quark propagator is of great importance, if the propagators exhibit singularities within the corresponding parabola, thus hampering the numerical procedure of solving the tBS equation. On the other side, the knowledge of the nature of singularities and their exact location in the complex plane will allow one to develop effective algorithms adequate for numerical calculations. For instance, if one determines exactly the domain of analyticity of the propagator functions, one can take advantage of the fact that any analytical function can always be approximated by rational complex functions [28]. Then, one can parametrize the integrand in the tBS equation by simple functions which will allow to carry out some integrations analytically. Such parametrisations have been suggested in Ref. [29] for meson masses $M<1$ $\mathrm{GeV}$ for which the propagator functions have been found to be analytical. Unfortunately, as shown in Ref. [21], for larger meson masses the propagator functions exhibit singularities within the domain of tBS integration and, as a consequence, parametrizations by rational functions are not possible. Nevertheless, even in this case, if the propagator functions have only isolated poles with known locations and residues, then calculations can be significantly simplified by splitting the singular functions into two terms, one being analytical in the considered region and the other one having a simple pole structure, as discussed below.

In Euclidean space the quark propagator obeys the truncated Dyson-Schwinger equation

$$
S^{-1}(p)=S_{0}^{-1}(p)+\frac{4}{3} \int \frac{d^{4} k}{(2 \pi)^{4}}\left[g^{2} \mathcal{D}_{\mu \nu}(p-k)\right] \gamma_{\mu} S(k) \gamma_{\nu},
$$

where $S_{0}^{-1}=i \gamma \cdot p+m_{q}$ and $m_{q}$ is the bare current quark mass. To emphasize the replacement of combined gluon propagator and vertex we use, as in Eq. (1), the notation $\left[g^{2} D_{\mu \nu}\right]$, where an additional power of $g$ from the second undressed vertex is included. For a consistent treatment of dressed quarks and their bound states, the dressed gluon propagator $\left[g^{2} \mathcal{D}_{\mu \nu}(p-k)\right]$ must be the same in the tBS (11) and the tDS equations (14). 


\section{B. Propagator functions}

The tDS equation (14) is a four dimensional integral equation in matrix form. Simplifications can be achieved in exactly the same manner as for the BS equation using the same spin matrices and the hyperspherical harmonics basis. Recall that the calculation of the renormalized Feynman diagrams leads to a fermion propagator depending on two functions, e.g. the renormalization constant $Z_{2}$ and the self-energy $\Sigma(p)$. Instead of $Z_{2}$ and $\Sigma(p)$ one can introduce other two quantities $A(p)$ and $B(p)$ or, alternatively, $\sigma_{s}(p)$ and $\sigma_{v}(p)$. In terms of these functions the dressed quark propagator $S(p)$ reads [6,, 31$]$

$$
S^{-1}(p)=i \gamma \cdot p A(p)+B(p), \quad S(p)=-i \gamma \cdot p \sigma_{v}(p)+\sigma_{s}(p)
$$

with

$$
\sigma_{v}(p)=\frac{A(p)}{p^{2} A(p)^{2}+B(p)^{2}}, \quad \sigma_{s}(p)=\frac{B(p)}{p^{2} A(p)^{2}+B(p)^{2}} .
$$

The resulting system of equations to be solved is a system of one-dimensional integral equations with respect to $A(p)$ and $B(p)$ (for details, see [14, 21]), which we solve numerically.

Independent parameters are $\omega, D$ and $m_{q}$. We find that the iteration procedure converges rather fast and practically does not depend on the choice of the trial start functions for $A(p)$ and $B(p)$.

In our subsequent analysis we employ the effective parameters from Refs. [18, 20], $\omega=0.5$ $\mathrm{GeV}$ and $D=16 \mathrm{GeV}^{-2}$.

\section{Solution in the right hemisphere, $\operatorname{Re} \tilde{p}^{2}>0$}

Evidently, the functions $A(p)$ and $B(p)$ are real at real values of $\tilde{p}^{2}$. However, since in the tBS equation the domain of definition of the propagator functions is complex, one needs an analytical continuation of the solution from the real positive axis $R e \tilde{p}^{2}>0$ to the whole complex domain inside the corresponding parabola. In our calculations the parabolic integration domain for solving the tBS equation we conveniently divide into two parts: (i) one (infinite) region where Re $\tilde{p}^{2}>0$, and (ii) a second one where Re $\tilde{p}^{2}<0$, which is restricted by the meson mass $M$, i.e. with the minimum (negative) value $\operatorname{Re} \tilde{p}^{2}=-M^{2} / 4$.

From the tDS equation (14) it is explicitly seen that, in the right hemisphere, the integrals converge in the tDS equation. That means, an analysis of the behaviour of the solution for 
Re $\tilde{p}^{2}>0$ for large $\left|\tilde{p}^{2}\right|$ can be accomplished directly by means of the real solutions $A(p)$ and $B(p)$ obtained along the real axis in the tDS equation and by utilization subsequently the tDS equation with complex external momenta to compute $A(p)$ and $B(p)$ in the desired domain.

Another method of finding $A\left(\tilde{p}^{2}\right), B\left(\tilde{p}^{2}\right), \sigma_{s}\left(\tilde{p}^{2}\right)$ and $\sigma_{v}\left(\tilde{p}^{2}\right)$ in the complex plane is to solve the tDS equation once along a closed contour and then to use the Cauchy theorem

$$
A(z)=\frac{1}{2 \pi i} \oint \frac{A(\xi)}{\xi-z} d \xi
$$

to calculate the required quantities in any point inside the contour, see also see Ref. [19].

Then, by the same method one can compute the Cauchy integrals of $A\left(\tilde{p}^{2}\right), B\left(\tilde{p}^{2}\right), \sigma_{s}\left(\tilde{p}^{2}\right)$ and $\sigma_{v}\left(\tilde{p}^{2}\right)$ to check them for analyticity. We performed such an analysis and found that in the right hemisphere the Cauchy integrals for the solutions $A\left(\tilde{p}^{2}\right), B\left(\tilde{p}^{2}\right), \sigma_{s}\left(\tilde{p}^{2}\right)$ and $\sigma_{v}\left(\tilde{p}^{2}\right)$ for each type of quarks $(\mathrm{u}, \mathrm{d}, \mathrm{s}$ and $\mathrm{c})$ is zero, i.e. they are analytical functions of $\tilde{p}^{2}$. Consequently, the integral kernel $S_{\alpha \beta}(\tilde{p}, \tilde{k}, m, n)$ in Eq. (10) is finite in this case. According to the theory of Fredholm type equations, one can infer that such a kernel possesses a discrete spectrum of eigenvalues.

\section{Parametrization of propagator functions at $R e \tilde{p}^{2}>0$}

The interaction kernel and its eigenvalues can be found numerically by solving the tDS equation for $\sigma_{s, v}$ and by implementing this solution into the numerical procedure for tBS equation. In principle, one can avoid such cumbersome calculations of $S_{\alpha \beta}(\tilde{p}, \tilde{k}, m, n)$ by taking advantage of the analyticity of $\sigma_{s, v}$ in the right hemisphere. It is known [28] that for any analytical function one can find convenient parametrizations in terms of rational functions, which in turn can be chosen in such a form as to be able to calculate the hyperangular integrals explicitly.

A convenient choice for the parametrization to calculate the integrals over Euclidean momentum $k$ in Eq. (10) could be of the following form

$$
\sigma_{s, v}\left(\tilde{k}^{2}\right)=\sum_{i} \frac{\alpha_{i}(s, v)}{\tilde{k}^{2}+\beta_{i}^{2}(s, v)}+\sum_{i} \frac{\alpha_{i}^{*}(s, v)}{\tilde{k}^{2}+\beta_{i}^{* 2}(s, v)},
$$

where the complex parameters $\alpha_{i}$ and $\beta_{i}$ can be easily obtained by fitting the corresponding solution along the real axis of $\tilde{k}^{2}$. We use the Levenberg-Marquardt algorithm for fitting. With such a monopole form of the parametrization the corresponding integral in (10) can be reduced 
to a sum of integrals of the form

$$
\mathcal{I}_{m n}^{\lambda}(z)=\int_{-1}^{1} d \xi\left(1-\xi^{2}\right)^{\lambda-1 / 2} \frac{G_{n}^{\lambda}(\xi) G_{m}^{\lambda}(\xi)}{\xi-i z} ; \quad \text { with } \quad z \sim \frac{\operatorname{Re} \tilde{k}_{1,2}^{2}+\beta^{2}}{M k}
$$

which can be calculated explicitly (see Appendix, Eq. (A3) ).

We find that for each function in Eq. (18) the first three terms, which involve 12 parameters, are quite sufficient to obtain a good approximation of the solution. In Tables II and II we present the sets of parameters $\alpha_{i}(s, v)$ and $\beta_{i}(s, v)$ for $\mathrm{u} / \mathrm{d}, \mathrm{s}$ and c quarks, obtained for $\sigma_{s}$ and $\sigma_{v}$, respectively, from a fit in the interval $-0.15(\mathrm{GeV} / \mathrm{c})^{2}<\tilde{k}^{2}<10(\mathrm{GeV} / \mathrm{c})^{2}$. In spite of the quality of the excellent fit it should be noted, however, that since the parametrized functions are of a rather simple shape, the obtained sets of parameters $\left\{\alpha_{i}\right\}$ and $\left\{\beta_{i}\right\}$ are far from being unique, i.e. one can achieve a similar quality of the fit with many other choices of $\left\{\alpha_{i}\right\}$ and $\left\{\beta_{i}\right\}$. The only restriction is that the "mass" parameters $\left\{\beta_{i}\right\}$ must not provide singularities, neither along the real axis, nor in the complex plane inside the parabola, see also Ref. [33]. The obtained sets of parameters, Tables I and II, have been used in our calculations of the propagator functions at $\operatorname{Re} \tilde{k}^{2}>0$, where they are always analytical.

TABLE I: The parameters $\alpha_{i}(v)$ and $\alpha_{i}(s)$ for the effective parametrizations, Eq. (18), for three values of the bare quark mass.

\begin{tabular}{ccccc}
\hline$m_{q}[\mathrm{MeV}]$ & $\alpha_{i}$ & 1 & 2 & 3 \\
\hline 5 & $\alpha_{v}$ & $(0.1915,0.94311)$ & $(0.15360,-0.18621)$ & $(0.15360,-0.18621)$ \\
& $\alpha_{s}[\mathrm{GeV}]$ & $(0.03018,0.48657)$ & $(-0.042732,-0.77654)$ & $(0.015015,0.02499)$ \\
\hline \multirow{2}{*}{115} & $\alpha_{v}$ & $(0.18503,0.18389)$ & $(0.21166,-0.74513)$ & $(0.10244,0.22828)$ \\
& $\alpha_{s}[\mathrm{GeV}]$ & $(-0.20626,0.59157)$ & $(0.31850,0.025363)$ & $(-0.055932,0.085008)$ \\
\hline \multirow{2}{*}{1000} & $\alpha_{v}$ & $(-0.17206,-0.77962)$ & $(0.19591,-1.0314)$ & $(0.48176,0.51818)$ \\
& $\alpha_{s}[\mathrm{GeV}]$ & $(1.3803,0.94714)$ & $(-0.94419,-0.27285)$ & $(0.064465,0.071692)$ \\
\hline
\end{tabular}

\section{E. Solution of the tDS equation at $\operatorname{Re} \tilde{k}^{2}<0$}

At $\operatorname{Re} \tilde{k}^{2}<0$ we use either the Cauchy theorem in the domain where the propagator functions are still analytical or the prescription explained below, if there are singularities. In Ref. [21] has been found that at large values of the meson masses $M>1 \mathrm{GeV}$ and at $\operatorname{Re} \tilde{k}^{2}<0$ 
TABLE II: The parameters $\beta_{i}(v)$ and $\beta_{i}(s)$ for the effective parametrizations, Eq. (18), for three values of the bare quark mass.

\begin{tabular}{|c|c|c|c|c|}
\hline$m_{q}[\mathrm{MeV}]$ & $\beta_{i}[\mathrm{GeV}]$ & 1 & 2 & 3 \\
\hline \multirow{2}{*}{5} & $\beta_{v}$ & $(0.5483,0.19010)$ & $(1.1385,0.26394)$ & $(1.1385,0.26394)$ \\
\hline & $\beta_{s}$ & $(0.53454,0.17611)$ & $(1.4018,0.046165)$ & $(-1.1163,1.2918)$ \\
\hline \multirow{2}{*}{115} & $\beta_{v}$ & $(0.62143,0.23396)$ & $(1.1353,0.19534)$ & $(0.69444,0.47076)$ \\
\hline & $\beta_{s}$ & $(0.78697,0.38476)$ & $(1.5621,0.70148)$ & $(1.0018,0.86675)$ \\
\hline \multirow{2}{*}{1000} & $\beta_{v}$ & $(1.8920,-0.71540)$ & $(2.120, \quad 0.62609)$ & $(1.9272,0.64545)$ \\
\hline & $\beta_{s}$ & $(1.8724,0.59370)$ & $(1.8750,0.90215)$ & $(1.9035,0.97726)$ \\
\hline
\end{tabular}

the propagator functions $\sigma_{s, v}\left(\tilde{k}^{2}\right)$ are not longer analytical functions having an infinite number of pole-like singularities in this region, which in principle give rise to numerical problems in solving the tBS equation. Nevertheless, as it has also been shown such singularities turn out to be integrable in the tBS equation, provided their exact locations and corresponding residues are known. For an analysis of the analytical properties of $\sigma_{s, v}\left(\tilde{k}^{2}\right)$ we suggested the following procedure [21]:

(i) Choose a relatively large domain within the parabola (13), enclose it with a contour and compute the Cauchy integrals of $A\left(\tilde{k}^{2}\right), B\left(\tilde{k}^{2}\right)$ and the inverse part of the propagator functions $\Pi\left(\tilde{k}^{2}\right) \equiv\left(\tilde{k} A\left(\tilde{k}^{2}\right)\right)^{2}+B^{2}\left(\tilde{k}^{2}\right)$. Vanishing integrals will imply that these functions are analytical within the chosen contour.

(ii) Compute Rouché's integral ${ }^{1}$ of the function $\Pi\left(\tilde{k}^{2}\right)$. Since in the previous item $\Pi\left(\tilde{k}^{2}\right)$ has been found to be analytical, such an integral, according to Rouché's theorem, gives exactly the number of its zeros inside the contour.

(iii) Compute the Cauchy integral of the propagator functions $\sigma_{s, v}\left(\tilde{k}^{2}\right)$ which, if Rouché's integral of $\Pi\left(\tilde{k}^{2}\right)$ is found to be an integer positive number, clearly must be different from zero. Moreover, it would imply that $\sigma_{s, v}\left(\tilde{k}^{2}\right)$ have poles inside the contour and their Cauchy integrals provide the corresponding residues, necessary in further applications, see below

(iv) Shrink the area of the contour until the Rouché's integral becomes equal to one and continue to squeeze the contour, by keeping the value of the Rouché's integral unchanged, until

\footnotetext{
${ }^{1}$ Rouché's integral of an analytical complex function $f(z)$ on a closed contour $\gamma$ is defined as $\frac{1}{2 \pi i} \oint_{\gamma} \frac{f^{\prime}(z)}{f(z)} d z$.
} 
the location of the pole is found with the desired accuracy.

The integrals to be calculated are:

$$
\begin{aligned}
& \left.\oint_{\gamma}\left[\xi^{2} A^{2}(\xi)\right)+B^{2}(\xi)\right] d \xi^{2}=0, \\
& \frac{1}{2 \pi i} \oint_{\gamma} \frac{\left.\left[\xi^{2} A^{2}(\xi)\right)+B^{2}(\xi)\right]_{\xi^{2}}^{\prime}}{\xi^{2} A^{2}(\xi)+B^{2}(\xi)} d \xi^{2}=N_{z}, \\
& \frac{1}{2 \pi i} \oint_{\gamma} \sigma_{s(v)}\left(\xi^{2}\right) d \xi^{2}=\sum_{i} \operatorname{res}\left[\sigma_{s(v)}\left(\xi_{i}^{2}\right)\right] .
\end{aligned}
$$

We find that at $M<1 \mathrm{GeV}$ all the integrals (20)-(22) are zero, implying that the tDS solution $A\left(\tilde{k}^{2}\right)$ and $B\left(\tilde{k}^{2}\right)$ and the propagator functions $\sigma_{s, v}\left(\tilde{k}^{2}\right)$ are analytical within the parabola (13). At $M>1 \mathrm{GeV}$ the Cauchy integrals for $A\left(\tilde{k}^{2}\right), B\left(\tilde{k}^{2}\right)$ and $\Pi\left(\tilde{k}^{2}\right)$ are still zero, i.e. the inverse propagator $\Pi\left(\tilde{k}^{2}\right)$ is still analytical, while for $\sigma_{s, v}\left(\tilde{k}^{2}\right)$ the Cauchy integrals do not longer vanish. Moreover, Rouché's integrals of $\Pi\left(\tilde{k}^{2}\right)$ are found to be integer positive numbers which clearly indicates that $\sigma_{s, v}\left(\tilde{k}^{2}\right)$ have poles in this region. The number of poles in the initial contour is given by the value of Rouché's integral (21).

For numerical calculations it is extremely important to find, with a good accuracy, the position of the poles and the corresponding residues for $\sigma_{s}\left(\tilde{k}^{2}\right)$ and $\sigma_{v}\left(\tilde{k}^{2}\right)$. In such a case, if the complex valued functions $\sigma_{s, v}\left(\tilde{k}^{2}\right)$ have only isolated poles $\tilde{k}_{0 i}^{2}$ within a certain domain and are analytical along its closing contour $\gamma$, they can be represented as

$$
\sigma_{s, v}\left(\tilde{k}^{2}\right)=\widetilde{\sigma}_{s, v}\left(\tilde{k}^{2}\right)+\sum_{i} \frac{\operatorname{res}\left[\sigma_{s, v}\left(k_{0 i}^{2}\right)\right]}{\tilde{k}^{2}-k_{0 i}^{2}},
$$

where $\widetilde{\sigma}_{s, v}\left(\tilde{k}^{2}\right)$ are analytical functions within the considered domain which can be computed as

$$
\widetilde{\sigma}_{s, v}\left(\tilde{k}^{2}\right)=\frac{1}{2 \pi i} \oint_{\gamma} \frac{\widetilde{\sigma}_{s, v}(\xi)}{\xi-\tilde{k}^{2}} d \xi=\frac{1}{2 \pi i} \oint_{\gamma} \frac{\sigma_{s, v}(\xi)}{\xi-\tilde{k}^{2}} d \xi .
$$

Such a representation (23) of the propagator functions in the presence of pole-like singularities allow to avoid numerical problems in calculations of the kernel (10). The product of two propagator functions in (10) in the presence of singularities receives the form

$$
\begin{aligned}
& \sigma_{1}\left(\tilde{k}_{1}^{2}\right) \sigma_{2}\left(\tilde{k}_{2}^{2}\right)=\tilde{\sigma}_{1}\left(\tilde{k}_{1}^{2}\right) \widetilde{\sigma}_{2}\left(\tilde{k}_{2}^{2}\right)+ \\
& +\widetilde{\sigma}_{1}\left(\tilde{k}_{1}^{2}\right) \sum_{j} \frac{\operatorname{res}\left[\sigma_{2}\left(k_{0 j}^{2}\right)\right]}{\tilde{k}_{2}^{2}-k_{0 j}^{2}}+\widetilde{\sigma}_{2}\left(\tilde{k}_{2}^{2}\right) \sum_{i} \frac{\operatorname{res}\left[\sigma_{1}\left(k_{0 i}^{2}\right)\right]}{\tilde{k}_{1}^{2}-k_{0 i}^{2}}+\sum_{i, j} \frac{\operatorname{res}\left[\sigma_{1}\left(k_{0 i}^{2}\right)\right] \operatorname{res}\left[\sigma_{2}\left(k_{0 j}^{2}\right)\right]}{\left(\tilde{k}_{2}^{2}-k_{0 j}^{2}\right)\left(\tilde{k}_{1}^{2}-k_{0 i}^{2}\right)},
\end{aligned}
$$


where $k_{0 i}^{2}$ and $k_{0 j}^{2}$ are the positions of the poles of the propagator functions $\sigma_{1,2}\left(\tilde{k}_{1,2}^{2}\right)$ of the first and second quark with the corresponding residues $\operatorname{res}\left[\sigma_{1}\left(k_{0 i}^{2}\right)\right]$ and $\operatorname{res}\left[\sigma_{2}\left(k_{0 j}^{2}\right)\right]$ in the integration domain of the tBS equation.

The first term in (25) is analytical everywhere within the integration domain of tBS and can be computed numerically by Eq. (24), while the last term in Eq. (25) has already an integrable form which, together with the Gegenbauer polynomials from (10), can be easily reduced to a sum of integrals like Eq. (19), see Appendix. The second and the third terms in (25) are still complex functions with singularities. However, the position of these singularities are exactly the same as found before, so that they can be again presented in the form (23) as a sum of analytical function and a pole-like structure and reduced to integrals of the type (19).

Equation (25) represents the main ingredient of our approach allowing to handle singularities in the tBS kernel (10).

\section{F. Location of singularities}

We calculated the integrals (20)-(22) for different current quark masses in the tDS equation (14) from $5 \mathrm{MeV}$, the mass corresponding to the light $\mathrm{u}$, d quarks, up to $1 \mathrm{GeV}$, which corresponds to the c quark. Results are presented in Fig. 1, where the location of first relevant poles is depicted for quarks with different current masses $m_{q}$, the values of which label the corresponding symbols in the figure. Also, the portions of the Euclidean space relevant to solve the tBS equation for bound states $M=1.1,1.5,2.0,2.5,3.0$ and $3.5 \mathrm{GeV}$ from left to right are presented as domains enclosed by correspondingly labeled parabolas (13).

Figure 1 allows, even prior to solve the tBS, for a rather general analysis of analytical properties of the meson bound states. For instance, it is seen that

(i) for meson masses $M<1 \mathrm{GeV}$ there are no singularities in the tBS kernel,

(ii) the first two self-conjugated poles appear at $M \sim 1 \mathrm{GeV}$ and belong to light $\mathrm{u}$, d quaks (the open diamonds in the figure),

(iii) the pole-structure of s quark propagators $\left(m_{q} \sim 115\right) \mathrm{MeV}$ starts at $M>1 \mathrm{GeV}$ and up to $M \sim 2.5 \mathrm{GeV}$ there are only two, self-conjugated poles. The third and forth poles for strange quarks are enclosed in parabolas $2.5 \mathrm{GeV}<M<3.0 \mathrm{GeV}$ and $3.0 \mathrm{GeV}<M<3.5 \mathrm{GeV}$, respectively,

iv) for the charmed quarks the singularity are located near $M \sim 3.5 \mathrm{GeV}$. 


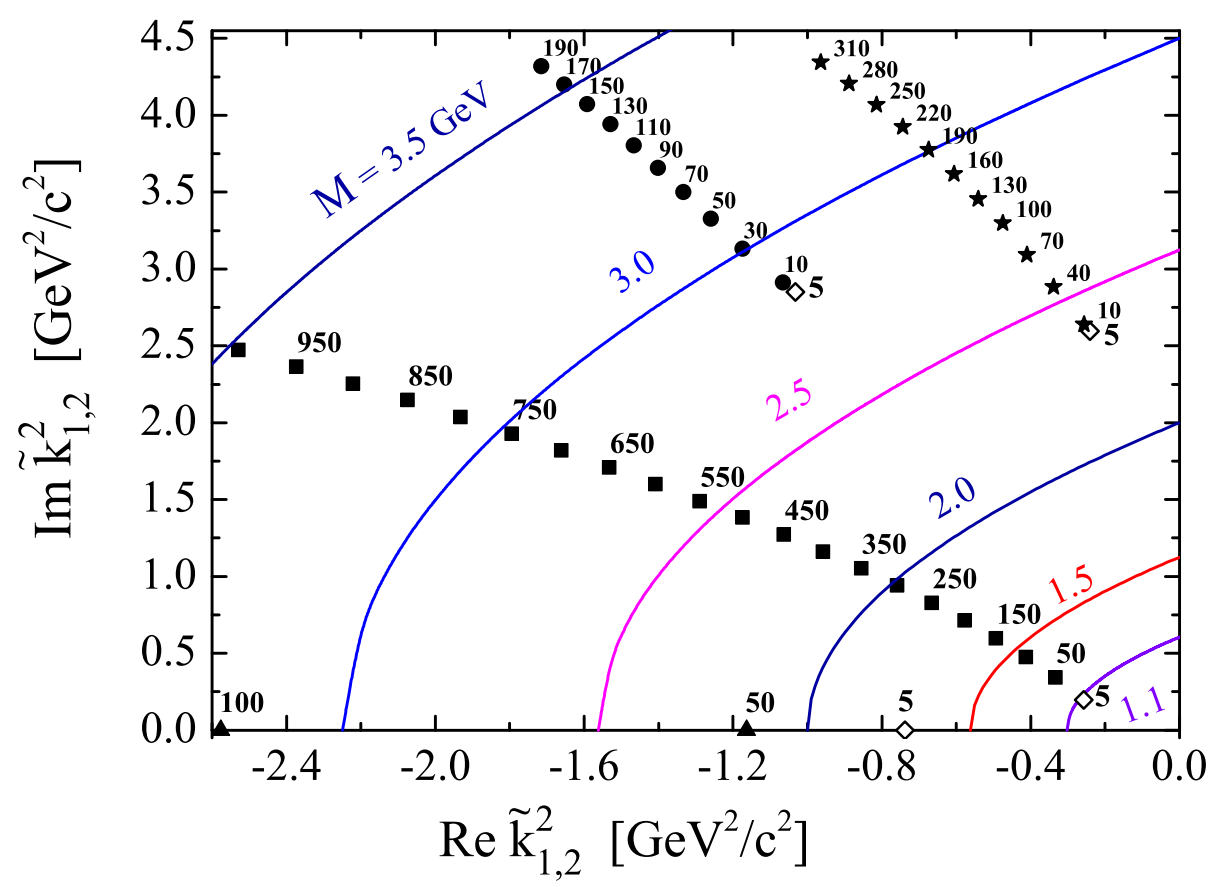

FIG. 1: (Color online) Positions of few first poles (full symbols) in the upper left hemisphere of the complex $\tilde{k}^{2}$ plane for various current quark masses (depicted in $\mathrm{MeV}$ ). The relevant sections of the parabola (13) corresponding to the meson bound-state mass $M$ are presented by solid curves for $M=1.1,1.5,2.0,2.5,3.0$ and $3.5 \mathrm{GeV}$, from right to left. The pole positions for a $5 \mathrm{MeV}$ quark are labeled by open symbols.

From this quick analysis one infers that calculations of mesons with masses $M \leq 1.1 \mathrm{GeV}$ (such as $\pi, \rho, K, \phi$ mesons) do not encounter difficulties related to singularities. For mesons with at least one light quark and $M \geq 1.1 \mathrm{GeV}$ (such as $D$ mesons, excited states of pions and kaons etc.) the tBS kernel contains propagator functions with singularities, which ought to be treated accurately, as explained above.

TABLE III: The position and residues of first few poles of propagator functions for $\mathrm{u}, \mathrm{d}$ quarks.

\begin{tabular}{llll}
\hline $\mathrm{i}$ & positions & $\operatorname{res}\left[\sigma_{s}\right]$ & $\operatorname{res}\left[\sigma_{v}\right]$ \\
\hline 1 & $(-0.2585,0.1958)$ & $\left(-1.613010^{-2},-0.5109\right)$ & $(0.2589,-0.8596)$ \\
2 & $(-0.2409,2.5947)$ & $\left(4.033610^{-2}, 0.1003\right)$ & $\left(2.338410^{-2}, 6.275010^{-2}\right)$ \\
3 & $(-0.7382,0.0)$ & $\left(6.886010^{-2}, 0.0\right)$ & $\left(-8.014710^{-2}, 0.0\right)$ \\
4 & $(-1.0415,2.8535)$ & $(-0.05,0.076)$ & $(0.0014,-0.052)$ \\
\hline
\end{tabular}


For completeness, in Table III we present the location of poles and their residue for the $\mathrm{u}, \mathrm{d}$ quark, relevant to most calculations of mesons at $1<M<3.5 \mathrm{GeV}$. Also it is worth to point out that the propagator functions for the s quark $\left(m_{q}=115 \mathrm{MeV}\right)$ possess only a self conjugated pole in the vicinity of the considered parabolas, located at $k_{01}^{2}=(-0.436 \pm 0.5131 i)(\mathrm{GeV} / \mathrm{c})^{2}$ with residues $\operatorname{res}\left[\sigma_{s}\right]=\left(9.0510^{-3} \mp 0.491 i\right) \mathrm{GeV}$ and $\operatorname{res}\left[\sigma_{v}\right]=0.261 \mp 0.538 i$ for $\sigma_{s}$ and $\sigma_{v}$, respectively. The second pole, located at $k_{02}^{2}=(-0.507 \pm 3.35 i)(\mathrm{GeV} / \mathrm{c})^{2}$ (with the respective residues $\operatorname{res}\left[\sigma_{s}\right]=\left(5.510^{-2} \pm 0.10 i\right) \mathrm{GeV}$ and $\left.\operatorname{res}\left[\sigma_{v}\right]=1.34,10^{-2} \mp 6.12,10^{-2} i\right)$, is located already too far from the corresponding parabola for strange mesons and, consequently is irrelevant in numerical calculations.

\section{NUMERICAL METHODS}

The performed analysis of quark propagators allows one to calculate the kernel of the tBS equation in the whole region of the complex Euclidean space relevant for the tBS equation. In numerical calculations we form the skeletons of approximate solutions and kernels by using the Gaussian method of computing integrals and by restricting the infinite sum over $n$ in Eqs. (7)-(9) by a finite value $M_{\max }$. The Gaussian quadrature formula assures a rather good convergence of the numerical procedure and provides the sought solution in the Gaussian nodes which are spread rather uniformly in the interval $0 \leq \tilde{p}<\infty$. In order to have the solution in detail at moderate values of $\tilde{p}$, which is the interval of the actual physical interests, one usually redistributes the Gaussian mesh making the nodes more dense at low values of $\tilde{p}$. To this end one applies an appropriate mapping of the Gaussian mesh by changing of variables as, e.g. in Ref. [25]. The resulting system of linear equations reads then as

$$
X=S X
$$

where the vector

$$
X^{T}=\left(\left[\left\{\varphi_{1}^{n}\left(\tilde{k}_{i}\right)\right\}_{i=1}^{N_{G}}\right]_{n=1}^{M_{\max }},\left[\left\{\varphi_{2}^{n}\left(\tilde{k}_{i}\right)\right\}_{i=1}^{N_{G}}\right]_{n=1}^{M_{\max }}, \ldots,\left[\left\{\varphi_{\alpha}^{m}\left(\tilde{k}_{i}\right)\right\}_{i=1}^{N_{G}}\right]_{n=1}^{M_{\max }}\right)
$$

represents the sought solution in the form of a group of sets of partial wave components $\varphi_{\alpha}^{n}$, specified on the integration mesh of the order $N_{G}$. The matrix $S$ is determined by the corresponding partial kernels (10), the Gaussian weights and the Jacobian of the mapping and is of the $N \times N$ dimension, where $N=\alpha_{\max } \times M_{\max } \times N_{G}$. Since the system of equations (26) is homogeneous, the eigenvalues of the bound state with mass $M$ is obtained from the condition 
$\operatorname{det}(S-1)=0$. Then, the partial components $\varphi_{\alpha}^{n}$ are found by solving numerically the system (26) at this bound-state mass $M$.

We use a combined method of finding the solution $X$. First, the Gauss-Jordan elimination and pivoting method involving the choice of the leading element is applied. Then the obtained solution is used as a trial input into an iteration procedure to find (after 5-10 iterations) more refined results.

\section{A. Pseudo-scalar meson ground states results}

As an example of our numerical study we exhibit in Fig. 2 the energy of the lowest bound states of a hypothetical meson $q q_{x}$ consisting of one given quark $q$ with the mass known from the tDS equation, bound with a second quark $q_{x}$ for which the input bare mass $m_{x}$ is let to vary arbitrarily. The corresponding effective parameters have been chosen as mentioned above and the bare masses for $q$ correspond to $\mathrm{u}, \mathrm{d}$, s and c quarks, $q=\mathrm{u}$ (with $m_{u}=0.005 \mathrm{GeV}$, solid line), $q=\mathrm{s}\left(\right.$ with $m_{s}=0.115 \mathrm{GeV}$, dashed line) and $q=\mathrm{c}$ (with $m_{c}=1.0 \mathrm{GeV}$, dot-dashed line). This figure illustrates the whole mass spectrum of pseudoscalar mesons with masses up to $3 \mathrm{GeV}$. In obtaining stable results for masses above $1 \mathrm{GeV}$, the pole structure of the propagators of the corresponding $q$ and $q_{x}$ quark have been treated as explained above, cf. Eq. (25) and Fig. 1.

As mentioned, Fig. 2 encodes results of ground-state masses for known pseudo-scalar mesons below $3 \mathrm{GeV}$. So, if the $q_{x}$ quark corresponds to a c quark, then at the intersection of the vertical line $m_{x}=m_{c}\left(\approx 1 \mathrm{GeV} / \mathrm{c}^{2}\right.$ ) with the "ux" curve one obtains the $D$ meson (with the quark contents uc), with the "sx" curve the $D_{s}$ meson and with the "cx" curve the $\eta_{c}$ meson, respectively. It is worth noting that the "ux" curve crosses the $m_{u}=0.115 \mathrm{GeV}$ line roughly at the same value of $M$ as the "sx" curve crosses the $m_{u}=0.005 \mathrm{GeV}$ line, thus providing a check of consistency of the approach and, at the same time, describing correctly the lowest pseudoscalar us state corresponding to the $\mathrm{K}$ meson. It can be seen that even without a fine tuning of $m_{s, c}$ the meson mass spectrum is reproduced fairly well: $135 \mathrm{MeV}$ ( $\pi^{0}$ meson), $497 \mathrm{MeV}$ ( $K$ meson), $1870 \mathrm{MeV}\left(D^{ \pm}\right.$meson), $1970 \mathrm{MeV}\left(D_{s}^{ \pm}\right.$meson) and $2980 \mathrm{MeV}\left(\eta_{c}\right.$ meson$)$.

\section{B. Vector meson states}

As mentioned above, the minor difference in calculations of pseudo scalar and vector meson masses consists in the fact that the basis of the spin angular harmonics (5) in the latter case 


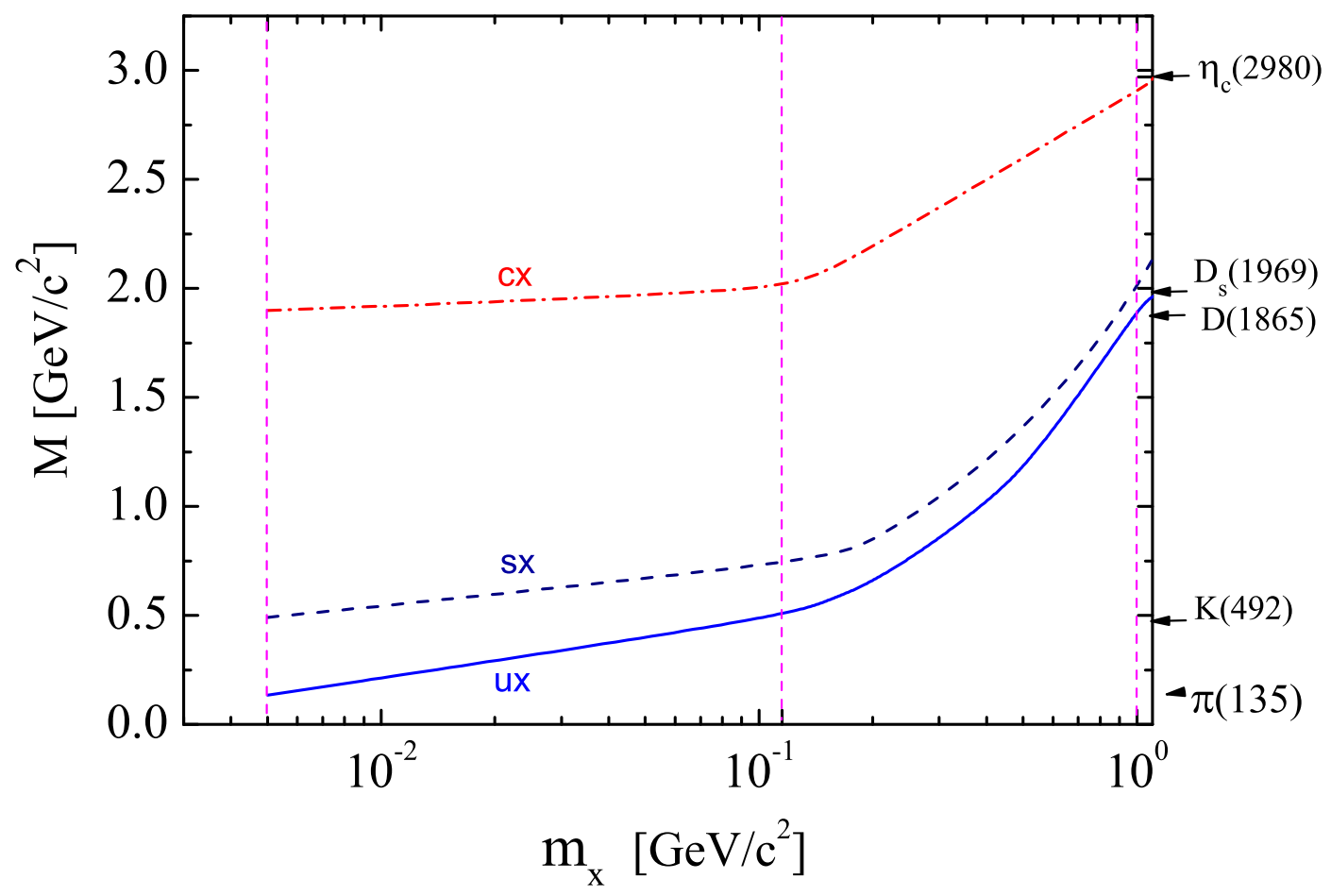

FIG. 2: (Color online) The bound-state masses (ground states) of a system $q q_{x}$ as a function of the bare mass $m_{x}$, for $m_{q}=0.005 \mathrm{GeV}$ (the curve labeled as "ux") or $m_{q}=0.115 \mathrm{GeV}$ (the curve labeled as " $s x "$ ) or $m_{q}=1.0 \mathrm{GeV}$ (the curve labeled as " $c x "$ ). The effective parameters of the vertex-gluon kernel (2) are $\omega=0.5 \mathrm{GeV}$ and $D=16 \mathrm{GeV}^{-2}$. The masses of the pseudoscalar $\pi, K, D, D_{s}$ and $\eta_{c}$ mesons according to [35] are indicated at the right side. The vertical dashed lines mark the selected bare quark masses for $\mathrm{u}, \mathrm{s}$, and $\mathrm{c}$.

has eight components instead of four in the former case. The general structures of the tBS kernel $S_{\alpha \beta}(\alpha, \beta=1 \ldots 8)$, Eq. (10), and of $A_{\alpha \beta}$, Eq. (12), remain the same. The propagator functions $\sigma_{s, v}$, being the solutions of the tDS equation, do not depend on the meson spin and are as before. That means that the present approach allows to perform, in the same manner, calculations of mass-spectra of mesons of any spin (scalar, pseudo-scalar, vector etc.), cf. [26]. Here it is worth emphasising that, as one can infer from Fig. 1, the propagator functions $\sigma_{s, v}$ do not contain singularities for vector mesons with ground-state masses up to $3.5 \mathrm{GeV}$ (such as $\rho, \phi, J / \Psi$ mesons). Consequently, all numerical calculations can be safely performed as in the previous approaches, cf. [11, 14, 26], without accounting for singularities. Likewise in the 

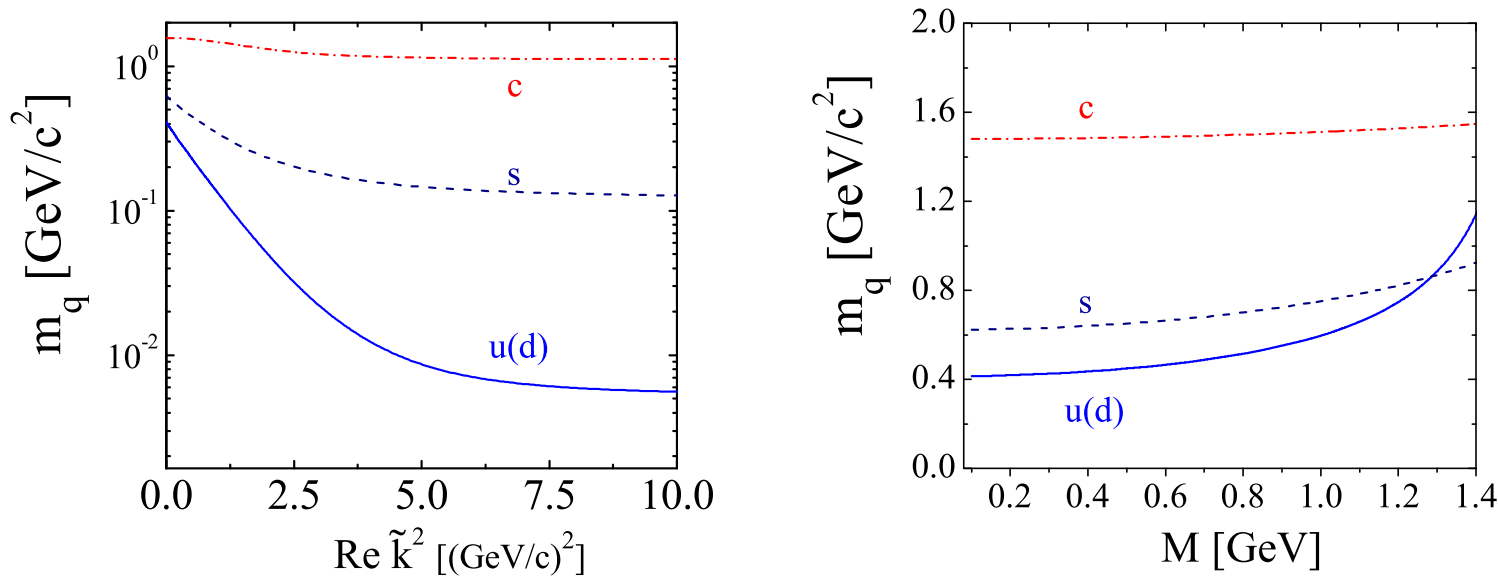

FIG. 3: (Color online) Dynamically dressed quark mass $m_{q}\left(\tilde{k}^{2}\right)=B\left(\tilde{k}^{2}\right) / A\left(\tilde{k}^{2}\right)$ from solutions of the Dyson-Schwinger equation along the real axis, i.e. for $\operatorname{Im}^{2}=0$. The left panel illustrates the dependence on $\operatorname{Rek}^{2}>0$, i.e. the behaviour of the solution of the tDS equation in real Euclidean space. In the right panel, the quark masses $m_{q}$ are calculated at the parabola vertex, $R e k^{2}=-M^{2} / 4$, and displayed as a function of $M$. A comparison of the two panels illustrates the dynamical mass increase in the complex Euclidean plane. Solid curves are for the light $\mathrm{u}, \mathrm{d}$ quarks, dashed curves for s quarks, and dot-dashed curves represent c quarks.

case of pseudo-scalar mesons, the performed analysis of the vector meson spectra shows that, results of numerical calculations are in an amazing good agreement with experimental data, cf. [11] .

\section{Excited meson states}

Within the present approach a description of radial excitations of mesons is straightforward. It suffices to find the next zeros of the determinant $\operatorname{det}(S-1)$ of the system (26) . It is worth noting that, in an analogy with the tBS equation for constituent quarks with constant masses, one would, at first glance, expect that the next zero of the determinant is to be searched for in a region of $M$ which dos not exceed the maximum value of masses of two dressed quarks. The tDS solution provides the corresponding maximum around $400 \mathrm{MeV}$ for the light $\mathrm{u}, \mathrm{d}$ quarks, $600 \mathrm{MeV}$ for s quarks and $1550 \mathrm{Mev}$ for c quarks, see Fig. 3, left panel. Correspondingly, one would expect that $u \bar{u}$ excited states cannot have a mass larger than $800 \mathrm{MeV}$. Analogous restrictions can be "deduced" for bound states with s and c quarks. 
However, in the non-Euclidean domain, which corresponds to $\tilde{k}_{1,2}^{2} \simeq-M^{2} / 4$, the dynamical quark masses $m_{q}\left(\tilde{k}^{2}\right)=B\left(\tilde{k}^{2}\right) / A\left(\tilde{k}^{2}\right)$ contributing to the tBS equation can strongly increase in dependence on $M$. This situation is illustrated in Fig. 3, right panel, where the dressed quark masses are depicted at the parabola vertex $R e \tilde{k}^{2}=-M^{2} / 4$ as a function of $M$. The corresponding masses $m_{q}\left(\tilde{k}^{2}\right)$ are larger in comparison with the net solution of the tDS equation, left panel. Hence, the Dyson-Schwinger equation allows to understand the formation mechanism of excited states which, from the constituent quark model point of view, can not be even predicted a priori. It should be emphasized that the quark masses $m_{q}$ in the complex Euclidean space have also singularities related to $A\left(\tilde{k}^{2}\right)=0$. If, in solving the tBS equation, instead of propagator functions $\sigma_{s, v}$ one deals with the quark mass as independent variable, an analysis of the singularities must be performed in detail prior solving the tBS and to find an adequate implementation in the numerical algorithm. It turns out that such an analysis becomes more involved than the one performed for the propagator functions presented above.

Coming back to calculations of the radially excited states, we mention that the determinant $\operatorname{det}(S-1)$ revealed its first excited $u \bar{u}$ state at $M \approx 1080 \mathrm{MeV}$, i.e. significantly above the maximum mass delivered by the Dyson-Schwinger equation alone at $R e \tilde{k}^{2}>0$. The third zero of the determinant for $u \bar{u}$ states has been found around $1300 \mathrm{MeV}$, corresponding to $\pi(1300)[35]$. Analogously, for the $c \bar{u}$ system, the first excited state is found to be around 2530 $\mathrm{MeV}$, which is in a good agreement with data, cf. [35]. Similar results have been obtained also by other groups, see e.g. Ref. [34].

\section{Exhausting method}

The above method of finding zeros of the determinant of the integral kernel is rather universal, provided the analytical properties of the kernel itself are known. However, the method becomes quite cumbersome if one tries to increase the accuracy of calculations by increasing the number $N_{G}$ of the Gaussian mesh and the number $M_{\max }$ of terms in the Gegenbauer decomposition. In this case, the dimension of the determinants drastically increases and the method of solving it for zeros becomes a challenging procedure. Another approach is to use an iteration method for solving the corresponding equations. As mentioned above, a finite kernel of a Fredholm type equation has a discrete and real spectrum with a nondecreasing sequence of eigenvalues $\lambda$. Moreover, it can be proven that, if such an equation is solved by iterations, 
it converges to the lowest value of the spectrum, i.e. to the ground state of the equation. It means that one can solve the BS equation as an eigenvalue problem,

$$
X=\lambda(M) S X
$$

for the eigenvalue $\lambda(M)$ as a function of the bound state mass. Then, the sought solution $M^{g . s .}$ can be found at $\lambda\left(M^{\text {g.s. }}\right)=1$. Then the kernel can be modified for the use of a iteration scheme for exited states. To this end, once the first eigenvalue is found, one constructs from this solution and from the old kernel a new one by subtracting from the previous kernel the contribution of its ground state. It is possible to do it in such a way that the new kernel will have the same spectrum of eigenvalues as the previous one except the eigenvalue already found. Obviously, for the new kernel the iteration method provides its ground state, which actually is the first excited state of the previous kernel. By continuing this procedure for the next eigenvalues one can find all the desired excited states. Such a method is known as the exhausting (depletion) method and was reported in some details in Ref. [27]. It seems that a similar method has been recently employed in Ref. [26].

This method holds only if the integral kernel is finite. In the case when the kernel $S$ contains singularities through the propagator functions, one has to handle them accurately as to obtain the desired finite $S$. It should be noted that often in numerical calculations of the kernel (10) the singularities are "overlooked" by not too large integration meshes used to evaluate the two dimensional integrals in (10). It means that in replacing the continuum Euclidean domain of integration (enclosed by the corresponding parabolas) by discrete meshes, one can "jump" over singularities and perform calculations without any troubles. However, increasing the density of mesh points one can approach closely the singularities and, consequently, the stability of solution can be lost.

In Fig. 4 we illustrate such a case. If one uses, e.g., Gaussian meshes $(j, \lambda)$ to integrate over $\tilde{k}$ and $\chi_{k}$ then in the tBS the corresponding values of momenta of quarks will be $\tilde{k}_{1,2(j, \lambda)}^{2}=$ $-M^{2} / 4+k_{j}^{2} \pm i M \tilde{k}_{j} \cos \chi_{k, \lambda}$. In Fig. 4 these nodes are depicted as $R e \tilde{k}_{1,2}^{2}(j)=-M^{2} / 4+k_{j}^{2}$ by full triangle symbols and, at each $R e \tilde{k}_{1,2}^{2}(j)$ the angular integration is depicted by vertical dotted lines. In the picture we adopted $M=1.5 \mathrm{GeV}$. The left panel corresponds to a 32 nodes Gaussian mesh for $\operatorname{Re} \tilde{k}_{1,2}^{2}$, while the right panel corresponds to a 96 nodes Gaussian mesh. As mentioned above, in our calculations we use a mapping procedure $k_{j}=k_{0} \frac{1+x_{j}}{1-x_{j}}$ with $k_{0}=0.1$, where $x_{j}$ are the Gaussian nodes defined from -1 to +1 . For illustration, the pole position of a $5 \mathrm{MeV}$ quark is depicted by full stars. It can be inferred from the picture that 

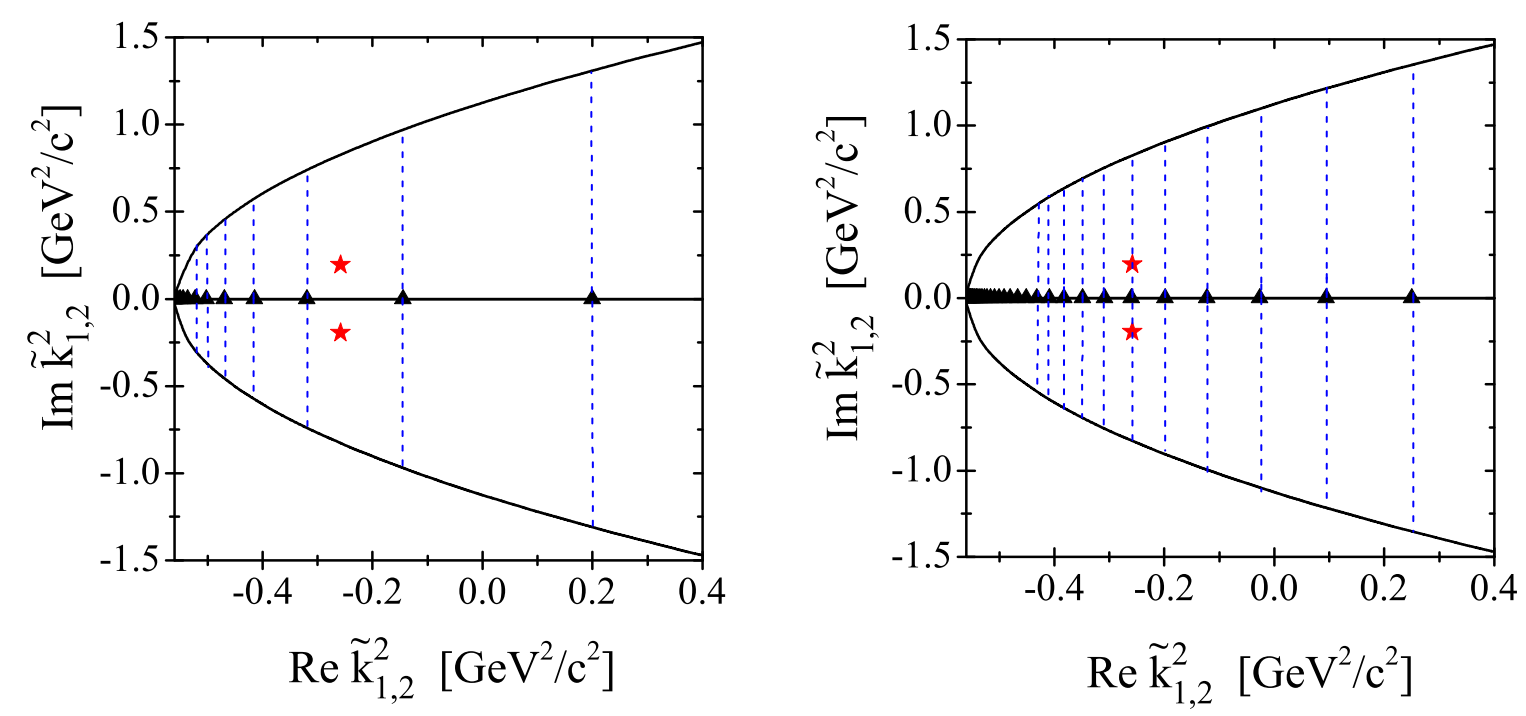

FIG. 4: (Color online) The interaction domain of the tBS equation over the complex $\tilde{k}_{1,2}^{2}$ plane which is the surface of parabola $\operatorname{Im} \tilde{k}_{1,2}^{2}= \pm M \sqrt{M^{2} / 4+R e \tilde{k}_{1,2}^{2}}$ for $M=1.5 \mathrm{GeV}$. The two selfconjugated poles for $\mathrm{u}, \mathrm{d}$ quarks are represented by stars. The full triangle symbols in left and right panels represent the Gaussian mesh for $R e \tilde{k}_{1,2}^{2}$ with 32 (left) and 96 (right) nodes, respectively, for the $d \tilde{k}$ integration which actually extends to $+\infty$. For each value of the node $R e \tilde{k}_{i}^{2}$ the angular integration $d \cos \chi_{k}$ goes along the vertical dashed lines (only a few ones are exhibited) corresponding to $-1 \leq \cos \chi_{k} \leq+1$.

in a numerical procedure all troubles can occur when the corresponding vertical line (angular integration) crosses the poles. At low values of Gaussian mesh the poles remain untouched (left panel) and the results could be finite without any additional treatment of the kernel (10). By increasing the Gaussian mesh the probability to meet the poles increases (right panel). In this case the tBS equation cannot be reliably solved numerically.

In principle, to avoid these problems one can use also the so-called "extrapolation" method, by calculating the dependence $\lambda(M)$ for masses $M<1 \mathrm{GeV}$, for which the kernel is an analytical function, then to extrapolate $\lambda(M)$ in the region with poles, see Ref. [26]. This method, if used not too far beyond the region of analyticity of the corresponding propagators, provides also rather good agreements with experimental data. 


\section{E. Equal quark masses, normalization condition}

A particular situation occurs in case of mesons with $M>1 \mathrm{GeV}$ formed by quarks of equal masses, e.g. in calculations of the excited states of pions above $1 \mathrm{GeV}$ or in calculating the normalization of the tBS partial amplitudes with at least one quark with poles, i.e. in calculations one meets products of two identical propagators with singularities. In such a case one has $k_{1}^{2}=k_{2}^{* 2}$ and, due to self-conjugated nature of the propagator functions in Eq. (25), one has also $\sigma_{2}\left(k_{2}^{2}\right)=\sigma_{1}^{*}\left(k_{1}^{2}\right)$. Moreover, the poles coincide for both propagators and in the sums over poles in (25) one also encounters the situation with $k_{0 i}^{2}=k_{0 j}^{* 2}$. In this case, i.e. the $R e \tilde{k}_{1,2}^{2}$ exactly coincides with the $R e k_{0 i}^{2}$ in the integrand (see right panel in Fig. 4), the angular integration reduces to (see Appendix, Eq. (A2)).

$$
\mathcal{I}_{m n}^{\lambda}(y) \sim \int_{-1}^{1} d \xi\left(1-\xi^{2}\right)^{\lambda-1 / 2} \frac{G_{m}(\xi) G_{n}(\xi)}{(\xi-y)^{2}},
$$

where $y$ is purely real and depends on the position of the pole, $y=\operatorname{Im} k_{0 i}^{2} / M \tilde{k}$. Since the pole is supposed to be inside the parabola one always has $|y|<1$. For such values of $y$, the integral (29) is not accessible in quadratures. Obviously, this situation is accidental and occurs only because of our specific choice of the Jacobi coordinates $k_{1}$ and $k_{2}$ when both quarks carry equal portions of the total momentum $P$ of the bound state. The problem can be solved by redistributing the total momentum $P$ between quarks as

$$
k_{1}=\eta P+k, \quad k_{2}=(1-\eta) P-k
$$

with $\eta \neq 0.5$. In this case, the momenta of quarks are no longer mutually complex conjugated, and the corresponding expression becomes again integrable. In our calculations of excited states of the pion we slightly changed $\eta$ away from $\eta=0.5$ by $\sim 10 \%$ which allows us to perform safely the necessary calculations. In principle, the solution of the BS equation in the ladder approximation must be independent of $\eta$, see e.g. Ref. [36], which, in the rainbow approximation, was numerically confirmed in several papers cf. [8, 9, 29]. It should be noted, however, that in concrete calculations where the Gegenbauer expansion is truncated at some value $M_{\max }$ the numerical solution may depend on the choice of $\eta$. In our calculations we investigated the sensitivity of numerical solutions on $M_{\max }$ and found that for $\eta=0.5$ the solution of the tBS equation is stable already at $M_{\max }=4-5$. It remains stable also if we change $\eta$ by $\sim(5-10) \%$. Larger deviations of $\eta$ will require a reanalysis of the dependence on $M_{\max }$ 
A few comments about the normalization of the tBS amplitude are in order here. Since the BS equation is a homogeneous equation, the solution for the amplitude must be additionally normalized. The normalization condition in the general case has been derived in Ref. [4]. For our case it reads [16, 18]

$$
\frac{3}{2} \frac{\partial}{\partial P_{\mu}} \operatorname{Tr}\left[\int \frac{d^{4} k}{(2 \pi)^{4}} \bar{\Gamma}(-P, k) S\left(k_{1}\right) \Gamma(P, k) S\left(-k_{2}\right)\right]=2 P_{\mu} .
$$

In the ladder approximation, due to translation invariance the tBS amplitude, $\Gamma(P, k)$ does not depend on the total momentum $P$ and often the derivative $\partial / \partial P_{\mu}$ is moved inside the integral acting only on $S\left(k_{1,2}\right)$. Such an operation is mathematically correct only if the integral is absolutely convergent. If so, then the derivative $\partial / \partial P_{\mu}=\eta \partial / \partial k_{1 \mu}$ acting, e.g., on the first propagator $S\left(k_{1}\right)$, leads to an expression of the form

$$
\partial S\left(k_{1}\right) / \partial k_{1 \mu}=-S\left(k_{1}\right)\left[i \gamma_{\mu} A\left(k_{1}\right)+A^{\prime}\left(k_{1}\right)+B^{\prime}\left(k_{1}\right)\right] S\left(k_{1}\right)
$$

i.e. after calculations of the corresponding traces, the remaining integral will contain two identical propagators. Calculations of such integrals are cumbersome, but straightforward.

In the case of singularities, if one still interchanges the derivative with integration, the remaining integral after traces lead again to divergences of the type (29). In this case, since the two propagators refer to the same quark, even the use of $\eta \neq 0.5$ does not cure the problem. It merely implies that one cannot exchange derivatives and integrations if the integral is not absolutely convergent. To normalize correctly the tBS amplitude in the presence of poles in propagators, one must first calculate the corresponding traces in (31) as a function of $P_{\mu}$ and then evaluate the derivatives numerically.

\section{SUMMARY}

We analyse the truncated Dyson-Schwinger (tDS) and Bethe-Salpeter (tBS) equations in the Euclidean complex momentum domain which is determined by the mass $M$ of mesons as quark-antiquark bound states. Within the ladder rainbow truncation, only the infrared term in the combined effective vertex-gluon kernel is retained. The locations of singularities of the propagator functions and their residues are determined with high accuracy in the whole region relevant to describe mesons with energy (masses) up to $M \leq 3.5 \mathrm{GeV}$. We propose a method of separating the analytical part and the pole structure in the propagators to be further implemented easily in numerical algorithms. It is demonstrated that the part with singularities 
can be integrated explicitly, avoiding in such a way difficulties in handling numerically singular quantities. The proposed method has been applied to calculate the mass spectra of pseudoscalar mesons with $M \leq 3.5 \mathrm{GeV}$ with and without singularities in the propagator functions. We obtain a good agreement with experimental data.

The performed analysis is aimed at elaborating adequate numerical algorithms to solve the BS equation in presence of singularities and to investigate the properties of mesons, such as the open charm $D$ mesons, related directly to physical programmes envisaged, e.g. at FAIR. Then, as our ultimate goal, the performed analysis is to be used as a base line for investigations of mesons at finite temperatures and baryon densities.

\section{Acknowledgments}

This work was supported in parts by the Heisenberg - Landau program of the JINR - FRG collaboration, GSI-FE and BMBF O5P12CRGH1. LPK appreciates the warm hospitality at the Helmholtz Centre Dresden-Rossendorf. The authors gratefully acknowledge discussions with M. Lutz, M. Viebach, R. Williams T. Hilger and C. Fischer and thank T.E. Cowan for the support of our research project.

\section{Appendix A: Useful relations}

For the product of two propagator functions in (10) the pole part (25) can be rewritten as

$$
\begin{aligned}
& {\left[\frac{1}{\tilde{k}_{1}^{2}-k_{0 i}^{2}}\right]\left[\frac{1}{\tilde{k}_{2}^{2}-k_{0 j}^{2}}\right]=\left[\frac{1}{R e \tilde{k}_{1}^{2}+i M \tilde{k} \xi-k_{0 i}^{2}}\right]\left[\frac{1}{R e \tilde{k}_{2}^{2}-i M \tilde{k} \xi-k_{0 j}^{2}}\right]=} \\
& =\frac{1}{(M \tilde{k})^{2}}\left[\frac{1}{\xi-i\left(\tilde{R} e k_{1}^{2}-k_{0 i}^{2}\right) / M \tilde{k}}\right] \cdot\left[\frac{1}{\xi-i\left(-R e \tilde{k}_{2}^{2}+k_{0 j}^{2}\right) / M \tilde{k}}\right]= \\
& \left.=\frac{1}{\Delta z_{i j} M \tilde{k}}\left[\frac{1}{\xi-i z_{i}^{(1)}}-\frac{1}{\xi-i z_{j}^{(2)}}\right] a 1\right)
\end{aligned}
$$

where

$$
\operatorname{Re} \tilde{k}_{1}^{2}=\operatorname{Re} \tilde{k}_{2}^{2}=-M^{2} / 4-\tilde{k}^{2}, \quad z_{i, j}^{(1,2)}=\frac{ \pm R e \tilde{k}_{1,2}^{2} \mp k_{0 i, j}^{2}}{M \tilde{k}}, \quad \text { and } \Delta z_{i j}=z_{i}^{(1)}-z_{j}^{(2)} .
$$

Then the main integral in the tBS kernel (10) with pole-like singularities is 


$$
\begin{array}{r}
\mathcal{I}_{m n}^{\lambda}(z)=\int_{-1}^{1} d \xi\left(1-\xi^{2}\right)^{\lambda-\frac{1}{2}} G_{m}^{\lambda}(\xi) G_{n}^{\lambda}(\xi) \frac{1}{\xi-i z}= \\
-\frac{2 \sqrt{\pi}}{\Gamma(\lambda)}\left(\frac{1}{2}\right)^{\lambda-\frac{1}{2}} e^{\left(\frac{1}{2}-\lambda\right) i \pi}\left(-z^{2}-1\right)^{\frac{2 \lambda-1}{4}} G_{m i n}^{\lambda}(i z) Q_{\text {max }+\lambda-\frac{1}{2}}^{\lambda-\frac{1}{2}}(i z),
\end{array}
$$

where $G_{m}^{\lambda}(x)$ are the Gegenbauer polynomials obeying the following recurrent relations

$$
\begin{aligned}
& 2 \lambda\left(1-\xi^{2}\right) G_{n-1}^{\lambda+1}(\xi)=(2 \lambda+n-1) G_{n-1}^{\lambda}-n \xi G_{n}^{\lambda}(\xi) \\
& (n+1) G_{n+1}^{\lambda}(\xi)=2(n+\lambda) \xi G_{n}^{\lambda}-(n+2 \lambda-1) G_{n-1}^{\lambda}(\xi),
\end{aligned}
$$

$Q_{m}^{\lambda}(x)$ are the Legendre functions of the second kind and $\min =\min (m, n), \max =\max (m, n)$.

Some useful relations with Legendre functions related to computations of our integral are

$$
\begin{aligned}
& Q_{n+1 / 2}^{1 / 2}(i z)\left(-z^{2}-1\right)^{1 / 4}=-i^{n} \sqrt{\frac{\pi}{2}} Z^{n+1}, \\
& Q_{n+1 / 2}^{3 / 2}(i z)\left(-z^{2}-1\right)^{3 / 4}=-i^{n+1} \sqrt{\frac{\pi}{2}}\left(n z Z^{n+1}+(n+1) Z^{n}\right), \\
& Q_{n-1 / 2}^{3 / 2}(i z)\left(-z^{2}-1\right)^{3 / 4}=-i^{n} \sqrt{\frac{\pi}{2}}\left((n-1) z Z^{n}+n Z^{n-1}\right), \\
& Q_{n+1 / 2}^{5 / 2}(i z)\left(-z^{2}-1\right)^{5 / 4}=i^{n} \sqrt{\frac{\pi}{2}}\left(n(n-1) z^{2} Z^{n+1}+(n-1)(2 n+3) z Z^{n}+n(n+2) Z^{n-1}\right), \\
& Z=\left(z-\sqrt{z^{2}+1}\right)=\frac{-1}{z+\sqrt{z^{2}+1}} .
\end{aligned}
$$

[1] CBM Collaboration, http://www.gsi.de/fair/experiments/CBM/index_e.html.

[2] PANDA Collaboration, http://www-panda.gsi.de/auto/phy/_home.htm.

[3] Nuclotron-based Ion Collider fAcility, http://nica.jinr.ru/,

[4] N. Nakanishi, Suppl. Prog. Theor.Phys. 43, 1 (1969).

[5] P. Maris, C. D. Roberts and P. C. Tandy, Phys. Lett. B 420, 267 (1998).

[6] P. Maris and C.D. Roberts, Int. J. Mod. Phys. E 12, 297 (2003).

[7] R. Alkofer and L. Smekal, Phys. Rept. 353, 281 (2001).

[8] P. Maris and C. D. Roberts, Phys. Rev. C 56, 3369 (1997).

[9] P. Maris and P. C. Tandy, Phys. Rev. C 60, 055214 (1999).

[10] A. Holl, A. Krassnigg and C.D. Roberts, Phys. Rev. C 70, 042203 (2004). 
[11] M. Blank and A. Krassnigg, Phys. Rev. D 84, 096014 (2011);

M. Blank, A. Krassnigg and A. Maas, Phys. Rev. D 83, 034020 (2011).

[12] T. Hilger, C. Popovici, M. Gomez-Rocha, A. Krassnigg, e-Print: arXiv:1409.3205 [hep-ph].

[13] A. Krassnigg and P. Maris, J. Phys. Conf. Ser. 9, 153 (2005).

[14] S. M. Dorkin, T. Hilger, L. P. Kaptari and B. Kämpfer, Few Body Syst. 49, 247 (2011).

[15] S. -x. Qin, L. Chang, Y. -x. Liu, C. D. Roberts and D. J. Wilson, Phys. Rev. C 84, 042202 (2011);

S. -x. Qin, L. Chang, Y. -x. Liu, C. D. Roberts and D. J. Wilson, Phys. Rev. C 85, 035202 (2012)

[16] P. Tandy, Prog. Part. Nucl. Phys. 39, 117 (1997).

[17] D. Horvatic, D. Blaschke, D. Klabucar and A. E. Radzabov, Phys. Part. Nuc. 39, 1033 (2008).

[18] R. Alkofer, P. Watson and H. Wiegel, Phys. Rev. D 65, 094026 (2002).

[19] C. S. Fischer, P. Watson and W. Cassing, Phys. Rev. D 72, 094025 (2005).

[20] C. D. Roberts, V. S. Bhagwat, S. V. Wright and A. Holl, Eur. Phys. J. ST 140, 53 (2007).

[21] S.M. Dorkin, L.P. Kaptari, T. Hilger and B. Kämpfer, Phys. Rev. C89 034005 (2014).

[22] A. E. Dorokhov and S. V. Esaybegyan, Phys. Lett. B 712, 381 (2012); Phys. Rev. C89, 034005 (2014).

[23] N. Souchlas, [arXiv:nucl-th/1006.0942], Jun 2010.

[24] L. P. Kaptari, A. Y. Umnikov, S. G. Bondarenko, K. Y. Kazakov, F. C. Khanna and B. Kämpfer, Phys. Rev. C 54, 986 (1996).

[25] S. M. Dorkin, M. Beyer, S. S. Semikh and L. P. Kaptari, Few Body Syst. 42, 1 (2008).

[26] C. S. Fischer, S. Kubrak, and R. Williams, arXiv:1406.4370 [hep-ph].

[27] S. M. Dorkin, L. P. Kaptari, C. C. degli Atti and B. Kämpfer, Few Body Syst. 49, 233 (2011).

[28] J. L. Walsh, "Interpolation and Approximation by Rational Functions in the Complex Domain", AMS Colloquium publications, v. XX, 1960.

[29] N. Souchlas, J. Phys. G, 37, 115001 (2010).

[30] C. S. Fischer, A. Maas and J. M. Pawlowski, Ann. Phys. 324, 2408 (2009).

[31] C. D. Roberts, Prog. Part. Nucl. Phys. 61, 50 (2008).

[32] N. Souchlas, Phys. Rev. D81, 114019 (2010).

[33] M. Bhagwat, M. A. Pichowsky and P. C. Tandy, Phys. Rev. D67 054019 (2003).

[34] A. Krassnigg, Phys. Rev. D 80, 114010, 2009

[35] K. Nakamura et al., J. Phys. G 37, 075021 (2010).

[36] C. Itzykson and J-B. Zuber "Quantum Field Thoery", McGraw-Hill, Book Company, 1980 